\title{
Image Watermarking: An evolution to content
}

\section{based approaches}

\author{
P. Bas, J-M. Chassery*and B. Macq \\ L.I.S. Laboratoire des Images et des Signaux, BP. 46, Saint Martin d'Heres, 38402, France
}

Laboratoire Tele, Batiment Stevin, Place du Levant, B-1348 Louvain-la-Neuve, Belgium

${ }^{*}$ Corresponding author: E-mail: Jean-Marc.Chassery@inpg.fr; Tel: (33) 04768262 63, Fax: (33) 0476826384 


\section{Summary}

A numerical medium has two major drawbacks; it is volatile and can be easily processed. The goal of watermarking is to insert a non-noticeable mark inside a numerical document to permit adding functionalities such as copyright prevention, document authentication or database retrieval.

Our study is concentrated on the field of image watermarking and the evolution from pixel-based approach to content-based methods is presented. We analyse watermarking constraints inherent to image processing and industrial applications. We bring a classification between additive, substitutive, frequential and spatial methods.

The use of image content permits to improve the robustness and invisibility of a marking scheme. Marking schemes can exploit the masking effect of the human visual system to improve the power of the mark without noticeable effects. If the content of the image is shifted by a geometrical transformation, the mark cannot be recovered without using a synchronization step. We present a brief overview of the existing self-synchronizing schemes that permit to recover the mark after geometrical attacks.

We have developed an original approach that exploit the content of the image and provide necessary references that permits the synchronization of the mark after geometrical transformations. A feature point detector is used to provide a set on points inside the image. The detector is based on the improvement of the Harris detector. We perform a triangular tessellation and the image is decomposed into a set of 
distinct triangles. Each triangle is marked independently. If the image undergoes a geometrical transformation, a sufficient number of triangles follows the transformation and the detection of the mark can be consequently be done. We have tested our algorithm with many processing such as rotations, scaling, jpeg compression, the mark removing attack Stirmark and the "print and scan" processing. The degree of robustness of our scheme depends of the content of the image. A discussion about our different results is made as a conclusion. 


\begin{abstract}
The paper is focused on the evolution of the watermarking schemes from pixel-based approaches to content based methods. Firstly the field of watermarking is presented, its implication in the real word is outlined. The second section describes a brief overview of different approaches in the spatial and the frequential domain. We then present different content-based approaches used to improve the invisibility or the synchronisation of the mark. The last section describes our content-based scheme, the synchronisation of the mark after image processing lies on the use of feature point detectors. The image is decomposed into a set of triangles and each triangle is individually marked.
\end{abstract}

Keywords: Watermarking, image content, geometrical transforms, feature points, triangular tessellation, additive scheme

\title{
2 Introduction
}

Information plays an important role in regards with physic for measurement (sensors), in regard with mathematics for modelling, in regard with signal and image processing and finally in regard with telecommunications techniques for exchange process.

Information needs protection protocols. Digital supports as CdRom and DVD can contain a huge amount of information of great quality. Nevertheless a numerical media has two major drawbacks: it is volatile and it can be easily processed. Consequently numerical content may be copied, modified or converted in other for- 
mats in an easy way.

Various techniques have been developed to protect the numerical media content. These techniques are well known under terms of Ciphering, Steganography and Watermarking.

- Ciphering

It consists to transform a message in a not accessible one. To read the cyphered message you need a key and the use of the corresponding program [1].

- Steganography

It consists to dissimulate a message in another one. This message may be a public one. The message is extracted using addresses of different parts of the supporting message [2].

- Watermarking

It consists to dissimulate a mark using a key in a public data. The mark needs to be undetectable and robust to techniques of information processing [3]. 


\section{Watermarking: generalities}

\subsection{Watermarking: definition and constraints}

In opposition with ciphering and steganography, watermarking consists in adding a mark, called watermark in a document. Such mark has to satisfy the following conditions:

The watermark must be undetectable by a non-authorized user.

The watermark process will satisfy conditions of visual quality. The marked image should not be perceptually different from the original.

The watermark should be robust to various processing like compression, quantization, D-A / A-D conversion, filtering and geometric transformations such as cropping, translation and rotations.

The watermark should contain a message. First techniques were devoted to detect presence or absence of a single mark [4]. More recently, developments are focused to insert a full message in the image [5] [6].

\subsection{Watermarking activities:}

Watermarking techniques are applied for lot of various activities illustrated on figure

1. The main functionalities of watermarking focus:

- The problem of authentication or integrity[7][8]. In this case the mark had to certify that the marked document is an original one. 
- The problem of copyright [9]. The mark permits to indicate the intellectual property of the document.

- The problem of database retrieval. The mark can contain a description of the content of the image.

All these information need different approaches of watermarking with various levels of complexity. For example, marks may be superposed in order to offer different functionalities (owner mark, editor mark, provider mark).

\subsection{Watermarking and applicative domains}

Since Internet diffusion, it is evident that watermarking is of wide interest. Audio and video documents need to be protected in such free access environment. Ebuiseness will be a perfect illustration of watermarking development[10]. Political institutions and industrial partners have taken positions for watermarking if we recall such recent examples:

- October 1998: Bill Clinton signs a law about the protection of digital documents (Digital Millennium Copyright Act)[11]. 
- February 1999: Signature of a convention between IBM, NEC, Hitachi and Pioneer Electronic Corporation about development of a standard for video watermarking with applications to DVD systems[12].

- March 1999: ASCAP society (American Society of Compositors, Authors and Productors) has a project for digital music watermarking in relation with ARIS society[13].

\subsection{Watermarking and world activities}

Watermarking is widely developed as well in research laboratories and in industrial activities. Since 1993 (date of the first publication in watermarking) the number of publications increases and specific meetings or workshop are organized. The not exhaustive following list indicates some industries involved in such domain:

- SureSign Digital Watermarking, Signum Technologies (Great Britain)

- Ice Audio Electronic Watermarking (Great Britain)

- Thomson-CSF (France)

- Philips (Netherlands)

- Eikona mark, Alpha Tec Ltd (Greece)

- Aris Technology (US) 
- Giovanni Media Security, Blue Spike Inc. (US)

- Digimark Corp. (US)

- Dice Company (Japan)

- MediaSec Technologies LLC (US)

- JK-PGS Signal Processing Lab (Switzerland)

- Digital Copyright Technomigies (Switzerland)

- Tigermark, NEC (Japan)

\section{Watermarking schemes: a classification}

Classical watermarking schemes include two main steps: the embedding and the detection of the mark.

- The embedding step consists to insert a mark in the initial image. The insertion may be performed in any representation domain such as the DCT coefficients, the DFT coefficients and wavelets coefficients. The HVS (Human Visual System) may be exploited to improve the invisibility of the mark [14] [15]. This insertion algorithm is public. The confidentiality is granted by the key for the mark generator. 
There are two families of watermarking schemes. The additives schemes consider the mark as a signal that is added to the components of the image (cf 2). The substitutive schemes replace some attributes of the image by the mark (cf Fig 3).

- The detection step is performed on the marked image or on a transformation version. The detection step can be developed in two different ways: using the original image or not. The use of the original is less practical but can be used to recover the mark after specific attacks such as geometrical transforms [16] $[17]$

As for the embedding scheme, two categories of detection are developed. For the additive schemes the detection of the mark is based on the correlation between the signature and the marked signal. For the substitutive scheme the detection of the mark is based on the examination of the extracted coefficients. To improve the robustness, the decoding of the mark can use error correcting codes [18].

The two following sections will illustrate the current methods in the spatial and in the frequential domain.

\subsection{Spatial methods}

The spatial methods work on the image itself. They insert or modify some pixel values using a key. 


\subsubsection{Manipulation of less significant bits:}

Such a method is based on the use of quantization noise issued from numeration process of the image. This quantization noise is replaced by a signal, the watermark. Such signal may be a predictive noise [19] or a SBPA binary sequence [20][21]. These approaches have the main disadvantage to have high sensitivity to new quantization process.

\subsubsection{Patchwork modification:}

Another technique will consist to identify randomly n pairs of pixels $\left(a_{i}, b_{i}\right)$. Pixels $a_{I}$ will be increased by value 1 and pixels $b_{I}$ will be decreased by value 1 [9]. Such method has also been developed by Pitas and Kaskalis [22]. The main drawback is lack of robustness to JPEG compression.

\subsubsection{Coding by textured blocs:}

This method consists in replacing textured blocs of the image by other textured similar blocs. The detection is performed using correlation. This approach has been enhanced by Caronni with repetition of a geometric pattern in the image [23]. Detection is also performed by correlation. 


\subsubsection{Visual mark insertion:}

Voyatsis and Pitas proposed to insert an image in the initial one [24]. The image to be inserted needs to be smaller and with a lower dynamic than initial one. A crypted version of it is used based on a torique automorphism.

\subsection{Frequential approaches}

Taking into account the constraint of robustness in regard with compression, in particular JPEG, lot of methods has been developed in the coefficients issued from the DCT transform [25].

\subsubsection{JPEG based approach:}

Zhao and Koch proposed to choose some DCT 8x8 blocs to insert the mark [26]. Coefficients corresponding with intermediate frequencies are selected and modified using the key. The watermark appears like a noise. Such approach is note efficient for JPEG quality less than 50

\subsubsection{Spread spectrum approaches:}

Cox et al compute the DCT of the entire image and limit their process to low frequencies $[4][27]$. They modify the $\mathrm{n}$ frequencies of higher amplitude using the following equations: 


$$
v_{i}^{\prime}=v_{i} *\left(1+a * x_{i}\right)
$$

with:

$v_{i}^{\prime} \mathrm{DCT}$ coefficient of the marked image

$v_{i}$ DCT coefficient of the initial image

a coefficient of invisibility

$x_{i}$ coefficient issued from a gaussian distribution.

Such approach uses the fact that the more significant information is included in low frequencies. Any alteration of such low frequencies, like attacks, will alter the image and consequently modify its visibility (cf Figure4 and 5).

The extraction of the mark is performed in a reverse manner using initial image. The extracted sequence $\mathrm{x}$ ' $\mathrm{i}$ is compared with the initial one $\mathrm{x}$ i using a correlation criterion. Other approaches have been developed without the use of original image [28].

The DCT transform is not the alone used transform. Ruanaidh et al [29] worked on the Fourier transform. Kundur and Hatzinakos worked on wavelets coefficients [30].

\subsection{Insertion of a message in the mark.}

One main objective of a watermarking system should be to insert a message in the image. To do that J.J.K. O Ruanaidh et al [5] proposed to replace the gaussian noise 
by a pseudo random sequence using CDMA techniques issued from spread spectrum approaches in communications. The message is numerically coded and each number is used to generate a random sequence (cf Figure 6). The mark is obtained as the sum of these sequences and after detection process, the mark is reconstructed.

Hartung et Girot[31], Kalker et al[32] have presented additive approaches in the spatial domain to embed several bytes into a video sequences.

\section{Content based approaches}

Watermarking schemes designers noticed that the content of the image could be used to improve the invisibility and the robustness of a marking scheme.

\subsection{Methods based on the Human Visual System}

The idea is to understand watermarking as masking phenomena with constraints of non visibility [33]. Such notion is well known on research activities using Human Visual System Properties.

For example on the following figure 7 we illustrate how to use the masking effect for embedding the mark.

Such considerations were also included in the quantization tables of JPEG and a first approach was proposed by Watson in order to introduce notion of adaptive tables [Wat 93]. 
Recently Delaigle et al proposed a technique of insertion based on the concept of local energy of a narrow band. Such technique was based on the Gabor filters widely used in the Eye modelling [15].

\subsubsection{Content insertion based on similarities}

An original approach based on similarities issued from fractal coding process has been introduced by Bas et al [34][35][36]. The main idea consists to force some regions of the image to offer non-noticeable similarities with other areas of the images. Illustration is given on Figure 8. The detection of the mark is done searching similarities on selected areas.

\subsection{Content modifications and countermeasures}

\subsubsection{Lost of synchronization}

If a geometrical transform is applied to the marked image the detection of the mark may become impossible. This is due to the fact that classical detection schemes use the internal structure of the image, constituted by an array of pixels with a fixed origin, to synchronize the detection of the mark.

If the mark image undergoes a geometrical transformation, the internal reference of the image is consequently modified and the detection scheme based on this reference can not recover the mark. 
The Stirmark attack uses this property to defeat many existing watermarking schemes. The stirmark algorithm performs small geometrical transformations such as light translations and rotations in an imperceptible way (cf Figure 9). Consequently the mark is not removed but shifted.

\subsubsection{Overview of self-synchronizing schemes}

In this section, we present a brief overview of existing watermarking schemes that permit to recover the mark after geometrical transformations. These schemes can be divided in 4 categories:

- schemes using periodical insertion of the mark

- schemes based on an invariant transform

- schemes inserting templates to identify the geometrical transform

- schemes using the original image to identify the geometrical transform

Kalker et al use an additive scheme in the spatial domain to mark video sequences[32].

To recover the mark after translation, the mark present periodicity characteristics. The recovering of the mark is done examining the correlation inside a small portion of each frame.

Delannay and Macq present a similar scheme based on a signature having cycling properties, the shape of the basic pattern depend on a secret key [37] and the mark can be recovered from any location of the image. 
Hartung et al present a scheme robust to the Stirmark attack[38]. The signature is inserted periodically in image blocks and the detection is performed on each block using a correlation function with 4 parameters to model affine transforms.

Another periodic insertion scheme has been developed by Kutter[39]. Due to the presence of a periodic mark inside the image, the cross-correlation function of the image present peaks that permit to identify the transform.

Ruanaidh and Pun present a marking scheme based on the properties of the Fourier-melin transform[5]. This transform based on a log-polar mapping of the Fourier domain is invariant to scaling, translation and rotations. Lin et al present a scheme based on the same transform [40].

Pereira and Pun propose a synchronizing scheme by inserting locale peaks named templates in the DFT domain[41]. The identification of the affine transform is done searching the localization of the templates and comparing their positions with the initial positions.

Davoine et al uses the original image to balance the geometrical deformations induced by stirmark[17]. A triangular tessellation is applied on the different images, the vertex of each triangle are slightly moved to best fit the geometrical transformation.

Sun et al propose to use feature points of the marked and original images to identify 
the geometrical transformation[42].

Alghoniemy and Tewfik propose to extract important coefficient of the wavelet transform of the original and distorted image[16]. The identification of the affine transform is done examining the distribution of the selected coefficients.

\section{A new scheme based on image content}

The main idea is to use salient points of the image to provide necessary references for the self-synchronisation. The extracted feature points located around corner and edges of the image define a set of distinct triangles. The mark is inserted inside each triangle.

\subsection{Feature point extraction}

\subsubsection{Selected detector}

Several feature points detector used for computer vision and image database retrieval $[43][44][45]$ have been tested. We have selected the Harris detector, which preserve the maximum of feature points after a geometrical transformation. The Harris detector response is given by:

$$
R_{H}=A B-C^{2}-k(A+B)^{2}
$$

where $A, B$ and $C$ are defined by: 


$$
\begin{aligned}
& A=X^{2} \otimes w \\
& B=Y^{2} \otimes w \\
& C=(X Y) \otimes w \\
& X=I \otimes(-1,0,1) \approx \delta I / \delta x \\
& Y=I \otimes(-1,0,1)^{T} \approx \delta I / \delta y
\end{aligned}
$$

$w$ is a blur mask which permits to improve the robustness of the detector.

\subsubsection{Improvements}

To estimate the robustness of the detector, we have defined a function name score given by:

$$
\text { score }=\frac{N b_{\text {pre }}-\left(N b_{\text {new }}+N b_{\text {dis }}\right)}{N b_{\text {pre }}+N b_{\text {dis }}}
$$

where $N b_{\text {pre }}, N b_{\text {new }}$ and $N b_{\text {dis }}$ represent respectively the number of points preserved, created and disappeared during the geometrical transformation. The score is maximal when $N b_{d i s}=N b_{\text {new }}=0$.

Feature points are concentrated around corners, edges and textures. In fact theses points are considered as references. We need to control the density of such points inside the image. Consequently our detector uses a circular mask and segmentation around the middle of the mask to locate corners, we fix a criterion of local maximum:

$$
\left\{p_{i, j} \in \psi\right\} \quad / \quad \forall\left(i^{\prime}, j^{\prime}\right) \in V_{i, j}, f(i, j)>f\left(i^{\prime}, j^{\prime}\right)
$$


where $V_{i, j}$ represents the neighbourhood of the pixel $(\mathrm{i}, \mathrm{j})$.

This criterion permits to control the density of feature points but also improves greatly the robustness of detectors against geometric transformations ( see Figure $10)$.

We have observed that the constructed detector is not isotropic; the score is maximal when the rotation angle is near cardinal direction $(\mathrm{N}, \mathrm{S}, \mathrm{E}, \mathrm{O})$, but is minimal when the rotation angle is near diagonal directions (NE, NW, SE, SW). This is due to the anisotropy properties of the derivative functions and the blurring filter.

Consequently we take in account this property by intersecting two feature point detections. The set of feature points $F$ is given by:

$$
F=F(I) \cap r_{-\frac{\pi}{4}}\left(F\left(r_{\frac{\pi}{4}}(I)\right)\right)
$$

where $\{F(A)\}$ represents the set of feature points extracted from the image $A$ and $r_{\theta}$ represents a rotation of an angle $\theta$ from the centre of the image.

Using this detection, the robustness to rotation has been greatly improved. Figure 11 presents the improvement of using an "isotropic" detector on image "lena512". 


\subsection{Embedding}

The embedding of the mark uses the references provided by the feature point detectors. The set of point provide a tessellation that is linked to the content of the image. We use the Delaunay tessellation to decompose the image into a set of disjoint triangles. The embedding of the mark is done inserting a signature in each triangle of the tessellation.

The general embedding scheme is represented on the figure 12. Let us decompose this scheme into different steps:

1. A random sequence $T_{w}$ is generated, the shape of this sequence is delimited by a right-angled isosceles triangle of an arbitrary size (in our tests $64 \times 64$ ). A secret key generates the sequence. To be robust to positioning error of feature points, the random sequence is spread on $2 \times 2$ pixel blocs.

2. The detection of feature points using our improved Harris detector is applied to the image.

3. A Delaunay tessellation $T=\left\{T_{i}\right\}, 0 \leq i<N$ is performed using feature points of the image. The detection of the mark is performed in each triangle $T_{k} \in T$.

4. An affine transform is performed on $T_{w}$ to map the shape of $T_{k}$. The affine transform is oriented using the magnitude of each angle issued from $T_{k}$. We use a spline-cubic interpolation during the transformation to preserve the high frequencies of $T_{r}$. The triangle $T_{m}$ is then obtained. 
5. $T_{m}$ is multiplied by a visual mask. This mask is obtained filtering the image with a laplacian mask. $T_{p}$ is generated.

6. The marked triangle is obtained adding $T_{p}$ and $T_{k}: T_{i}=T_{p}+T_{k}$.

7. $T_{i}$ is substitued to $T_{k}$.

\subsection{Detection}

The detection process uses the result of the feature point detection and consequently performs a self-synchronisation of the mark. If the marked image undergoes a geometric transformation, the set of salient points mainly follows the transformation and several triangles are consequently conserved. The presence of the signature in a triangle is certified by a correlation criterion.

The detection scheme is illustrated on the figure 13. We now detail the different steps of the detection of the mark, the three first steps are identical to the insertion scheme:

1. $T_{w}$ is generated from a random sequence depending on a secret key.

2. The detection of feature points using our improved Harris detector is applied to the image.

3. A Delaunay tessellation $T=\left\{T_{i}\right\}, 0 \leq i<N$ is performed using feature points of the image. The mark is inserted in each triangle $T_{k} \in T$. 
4. Each triangle $T_{k}$ is warp into a right-angled isosceles triangle $T_{\llcorner}$of size $64 \times 64$.

5. From $T_{\mathrm{L}}$ we proceed a Wiener processing using the statistics of $T_{w}$ to eliminate to components provided by the original image. We obtain $\hat{T}_{w}$ :

$$
\hat{T}_{w}(i, j)=\frac{\operatorname{Var}_{l o c}\left[T_{w}\right](i, j)}{\operatorname{Var}_{l o c}\left[T_{w}\right](i, j)+\operatorname{Var}_{l o c}\left[T_{\llcorner}\right](i, j)}\left[T_{\llcorner}(i, j)-\operatorname{Moy}_{l o c}\left[T_{\llcorner}\right](i, j)\right]
$$

$\operatorname{Var}_{l o c}()$ represent the local standard variance and $M o y_{l o c}()$ the local mean considered for location $(i, j)$.

6. We calculate the correlation between $T_{w}$ and $\hat{T}_{w}$ :

$$
\operatorname{corr}\left(T_{w} ; \hat{T}_{w}\right)=\frac{\sum_{i, j} T_{w}(i, j) \hat{T}_{w}(i, j)}{\sqrt{\sum_{i, j} T_{w}^{2}(i, j) \sum_{i, j} \hat{T}_{w}^{2}(i, j)}}
$$

7. The detection of the mark can be performed to two levels. First it can be done comparing the magnitude of $\operatorname{corr}\left(T_{w} ; \hat{T}_{w}\right)$ with a threshold for each triangle. The threshold is depending of a false response probability. We represent the statistic law in a case of a false response by a normal distribution.

8. A complementary decision is obtained using the global sum of $\operatorname{corr}\left(T_{w} ; \hat{T}_{w}\right)$.

9. The final decision is obtained from the different detection results.

\subsection{Results}

We have tested the robustness of the presented scheme with four different images showing a large variety of content (see figure 19). The image "lena" is a contrasted 
image with well-defined corners and edges. The image "water" is an homogeneous image with low frequency components. The image "baboon" contains a mixing of textured and homogeneous areas. The image "tree" contains high textured regions. Our algorithm has been confronted with five treatments:

- Jpeg compression

- the Stirmark attack

- Rotation of the image

- Scaling

- the "Print and Scan" test

\subsubsection{Jpeg Compression}

The table 1 indicates the number of triangle in which the mark is detected and the result of global detection after Jpeg compression. We have selected two different quality factors, $50 \%$ and $75 \%$. The figure 14 represents the evolution of the tessellation on the "lena" image after a Jpeg compression, triangle detected having the mark are represented with a white center.

These results illustrate the fact that our scheme is robust to Jpeg compression up to a quality factor of $50 \%$ and for different variety of images.

\subsubsection{Stirmark}

The stirmark attack is composed by local geometrical transforms and hence cannot be represented by a global affine transform. The robustness to the stirmark3.0 attack 
is illustrated on table 2 and figure 15.

The use of our content dependent scheme permits to perform a self-synchronization stage to recover the mark after the stirmark attack.

\subsubsection{Rotation}

We have tested the robustness of the presented scheme with rotation of slight $\left(10^{\circ}\right.$, $20^{\circ}$ ) and important $\left(45^{\circ}\right)$ angles (cf Fig16). The table 3 represents the robustness of our scheme after rotation.

The results indicate that the scheme is more robust with small angles. This is partly due to the anisotropy of our feature point detector.

\subsubsection{Scaling}

The figure 4 illustrates the reaction of the presented scheme to scaling operations with a factor of 0.9 and 0.7 (cf Fig17).

The scheme is still robust with small scaling operations but we can notice that the mark cannot be detected for a factor of 0.7 with textured images ("tree" and "baboon").

\subsubsection{Print and Scan}

The "print and scan" processing is a drastic processing because it is composed with a modification of pixels value and light geometric transforms. We can also observe a scanning noise due to the $\mathrm{A} / \mathrm{D}$ conversion. Table 5 shows the reaction of the presented scheme to a print and scan process with a $300 \times 300$ grey-level printer and 
300ppi scanner. We have applied a basic scanning and a another scanning after a slight rotation of the sheet (cf fig18).

The results demonstrate that our method is robust to the print and scan processing treatment for content structured images. For the tree image, the feature point detector cannot preserve a adequate number of triangles.

\subsubsection{Discussion and perspectives}

The different results illustrate the fact that the robustness our marking scheme depends on the behaviour of the feature point detector. We have seen that on structured images, having heavy edges and corners, our watermarking scheme is robust to a great variety of processing such as Jpeg compression on geometrical distortions. On the other hand, if the content of the image admits textured regions (case of the image "tree") the robustness of the marking scheme is reduced. Consequently our future works will focus on improving the robustness of our feature point detector.

\section{Concluding Remarks}

We have presented the progression of the watermarking schemes from an array representation to a content representation of the image. The geometrical distortions induced by images editing lead to the development of self-synchronizing schemes. We introduce the use of feature point detectors to provide necessary landmarks. The presented scheme uses a triangular tessellation to insert an additive mark on each triangle. Our results show that the proposed scheme is robust to Jpeg compression up to a quality factor of $50 \%$, the Stirmark attack and slight rotation and scaling. 


\section{References}

[1] D. Welsh, Codes and Cryptography, Oxford Science Publications., 1988.

[2] A. P. Petitcolas, R. J. Anderson, and M. G. Kunh, "Information hiding-a survey," Proceedings of the IEEE 87, pp. 1062-1078, july 1999.

[3] F. Hartung and M. Kutter, "Multimedia watermarking techniques," Proceedings of the IEEE 87, pp. 1079-1107, july 1999.

[4] I. Cox, J. Killian, T. Leighton, and T. Shamoon, "Secure spread spectrum watermarking for multimedia," IEEE Transactions on Image Processing 6, pp. 1673-1687, Dec. 1997.

[5] J. J. K. Ó Ruanaidh and T. Pun, "Rotation, scale and translation invariant spread spectrum digital image watermarking," Signal Processing 66, pp. 303317, May 1998.

[6] M. Barni, F. Bartolini, A. D. Rosa, and A. Piva, "Capacity of the watermarkingchannel: How many bits can be hidden within a digital image?," in Proc. SPIE, pp. 437-448, Jan. 1999.

[7] M. P. Queluz, "Content-based integrity protection od digital images," in Proc. SPIE, pp. 85-93, Jan. 1999.

[8] D. Kundur and D. Hatzinakos, "Digital watermarking for telltale tamper proofing and authentication," Proceedings of the IEEE 87, pp. 1167-1180, july 1999. 
[9] D. G. W. Bender and Morimoto, "Techniques for data hiding," in Proc. SPIE, vol. 2420, p. 40, Feb. 1995.

[10] G. Voyatzis and I. Pitas, "The use of watermarks in the protection of digital multimedia products," Proceedings of the IEEE 87, pp. 1197-1207, july 1999.

[11] "Dvd audio copyright specs emerge." http://www.nytimes.com/library/tech/98/10 /cyber/articles/29wipo.html, October 1998.

[12] "Digital copyright agreement for video." http://www.nytimes.com/library/tech/99 /02/biztech/articles/17blue.html, February 1999.

[13] "Ascap expands its audio watermarking efforts." http://www.ascap.com/press/aris-030399.html, Marsh 1999.

[14] E. J. D. R. B. Wolfgang, C. I. Podilchuk, "Perceptual watermarks for digital images and video," Proceedings of the IEEE 87, pp. 1108-1126, july 1999.

[15] J.-F. Delaigle, C. De Vleeschouwer, and B. Macq, "Watermaking algorithm based on a human visual model," Signal Processing 66, pp. 319-336, May 1998.

[16] M. Alghoniemy and A. H. Tewfik, "Geometric distortion correction in image watermarking," in Proc. SPIE, pp. 82-89, Jan. 2000.

[17] F. Davoine, P. Bas, P.-A. Hebert, and J.-M. Chassery, "Watermarking et rsistance aux dformations gomtriques," in Coresa99, (Institut-Eurecom, Sophia Antipolis, France), June 1999. 
[18] J. R. Hernandez, J.-F. Delaigle, and B. Macq, "Improving data hiding by using convolutional codes and soft-decision decoding," in Proc. SPIE, pp. 24-47, Jan. 2000.

[19] K. Tanaka, Y. Nakamura, and K. Matsui, "Embedding secret information into a dithered multi-level image," in Proc, Military Communications Conference, pp. 216-220, IEEE, 1990.

[20] R. Schyndel, A. Tirkel, and C. Osborne, "A digital watermark," in Int. Conf. on Image Processing (ICIP), vol. 2, pp. 86-89, IEEE, (Austin, Texas), Novembre 1994.

[21] R. Wolfgang and E. Delp, "A watermarking technique for digital imagery: Further studies," in Int. Conf. on Imaging Science, Systems and Technology, (Las Vegas, Nevada), Juillet 1997.

[22] I. Pitas and T. Kaskalis, "Applying signatures on digital images," in Workshop on Nonlinear Signal and Image Processing, pp. 460-463, IEEE, (Neos Marmaras), June 1995.

[23] G. Caronni, "Assuring ownership rights for digital images," in Proc. of Reliable IT Systems, VIS'95, Viewveg Publishing Company, (Germany), 1995.

[24] G. Voyatzis and I. Pitas, "Chaotic mixing of digital images and applications to watermarking," in European Conference on Multimedia Applications, Services and Techniques (ECMAST), (Louvain-la-Neuve), Mai 1996. 
[25] A. Bors and I. Pitas, "Image watermarking using det domain constraints," in Proc. ICIP, vol. 3, pp. 231-234, (Lausanne), 1999.

[26] K. E. Zhao J., "Embedding robust labels into images for copyright protection," tech. rep., Fraunhofer Institute for Computer Graphics, Darmstadt, Germany, 1994.

[27] I. Cox, J. Killian, T. Leighton, and T. Shamoon, "Secure spread sprectrum watermarking for multimedia," tech. rep., Nec Research Institute, Princeton, NJ, USA, Octobre 1995.

[28] A. Piva, M. Barni, F. Bartolini, and V. Capellini, "Dct based watermark recovering without resorting to the uncorrupted original image," in Proc. ICIP, pp. 520-523, 1997.

[29] J. Ruanaidh, F. Boland, and O. Sinnen, "Watermarking digital images for copyright protection," in Proc. of The Electronic Imaging and Visual Arts Conf., (Florence), Fvrier 1996.

[30] D. Kundur and D. Hatzinakos, "Digital watermarking based on multiresolution wavelet data fusion," in Special Issue on Intelligent Signal Processing, IEEE, 1997.

[31] F. Hartung and B. Girod, "Watermarking of uncompressed and compressed video," Signal Processing VOL.66, pp. 283-333, mai 1998.

[32] T. Kalker, G. Depovere, J. Haitsma, and M. Maes, "A video watermarking system for broadcast monitoring," in Proc. SPIE, pp. 103-112, Jan. 1999. 
[33] I. Cox and M. Miller, "A review of watermarking and the importance of perceptual modeling," in Proc. SPIE Conf. on Human Vision and Electronic Imaging II, N. R. Institute, ed., vol. 3016, pp. 92-99, Fvrier 1997.

[34] P. Bas, J.-M. Chassery, and F. Davoine, "A geometrical and frequential watermarking scheme using similarities," in Proc. SPIE, pp. 264-272, Jan. 1999.

[35] P. Bas, J.-M. Chassery, and F. Davoine, "Using the fractal code to watermark images," in Proc. ICIP, vol. 1, pp. 469-473, (Chicago), 1998.

[36] P. Bas, J.-M. Chassery, and F. Davoine, "Self-similarity based image watermarking," in Proc. EUSIPCO, vol. 4, pp. 2277-2280, (Rhodes), 1998.

[37] D. Delanay and B. Macq, "Generalized 2-d cyclic patterns for secret watermark generation," in Proc. ICIP, (to be published), Sept 2000.

[38] F. Hartung, J. Su, and B. Girod, "Spread spectrum watermarking: Malicious attacks and counter-attacks," in Proc. of SPIE: Security and Watermarking of Multimedia Contents, vol. 3657, pp. 147-158, (San Jose CA), January 1999.

[39] M. Kutter, "Watermarking resisting to translation, rotation and scaling," in Proc. of SPIE: Multimedia systems and applications, vol. 3528, pp. 423-431, (Boston), November 1998.

[40] C.-Y. Lin, J. A. Bloom, I. J. Cox, M. L. Miller, and Y. Lui, "Rotation-, scale, and translation-resilient public watermarking ofr images," in Proc. SPIE, pp. 90-98, Jan. 2000. 
[41] S. Pereira and T. Pun, "Fast robust template matching for affine resistant image watermarking," in International Workshop on Information Hiding, vol. LNCS 1768 of Lecture Notes in Computer Science, pp. 200-210, Springer Verlag, (Dresden, Germany), 29 September -1 October 1999.

[42] Q. Sun, J. Wu, and R. Deng, "Recovering modified watermarked image with reference to originale image," in Proc. SPIE, pp. 415-424, Jan. 1999.

[43] C. Harris and M. Stephen, "A combined corner and edge detector," in 4th Alvey Vision Conf, pp. 147-151, 1988.

[44] J. D. C. Achard-Rouquet, E. Bigorgne, "Un detecteur de point carateristiques sur des images multispectrales. extension vers un detecteur sub-pixellique," in GRETSI 99, pp. 627-630, Sept. 1999.

[45] S. M. Smith and J. M. Brady, "Susan - a new approach to low level image processing," International Journal of Computer Vision 23, pp. 45-78, may 1997. 


\section{Tables}

\begin{tabular}{|c|c|c|c|c|}
\hline & lena & tree & water & baboon \\
\hline $75 \%$ : detected triangle & $17 / 66$ & $4 / 66$ & $12 / 67$ & $7 / 67$ \\
\hline $75 \%$ : global detection & $\mathrm{Ok}$ & $\mathrm{Ok}$ & $\mathrm{Ok}$ & $\mathrm{Ok}$ \\
\hline $50 \%$ : detected triangle & $7 / 68$ & $2 / 66$ & $2 / 64$ & $9 / 66$ \\
\hline $50 \%$ : global detection & $\mathrm{Ok}$ & $\mathrm{Ok}$ & Failure & $\mathrm{Ok}$ \\
\hline
\end{tabular}

Table 1: Jpeg compression robustness for two quality factors: $75 \%$ et $50 \%$.

\begin{tabular}{|c|c|c|c|c|}
\hline & lena & tree & water & baboon \\
\hline detected triangle & $23 / 66$ & $3 / 66$ & $21 / 65$ & $3 / 62$ \\
\hline global detection & $\mathrm{Ok}$ & $\mathrm{Ok}$ & $\mathrm{Ok}$ & $\mathrm{Ok}$ \\
\hline
\end{tabular}

Table 2: Robustness of the presented scheme to Stirmark3

\begin{tabular}{|c|c|c|c|c|}
\hline & lena & tree & water & baboon \\
\hline $10^{\circ}:$ detected triangle & $10 / 65$ & $2 / 65$ & $19 / 66$ & $2 / 63$ \\
\hline $10^{\circ}:$ global detection & Ok & Failure & Ok & Failure \\
\hline $20^{\circ}:$ detected triangle & $7 / 68$ & $1 / 63$ & $13 / 69$ & $2 / 65$ \\
\hline $20^{\circ}:$ global detection & Ok & Failure & Ok & Failure \\
\hline $45^{\circ}:$ detected triangle & $2 / 68$ & $0 / 65$ & $8 / 67$ & $1 / 66$ \\
\hline $45^{\circ}:$ global detection & Failure & Failure & Ok & Failure \\
\hline
\end{tabular}

Table 3: Robustness to rotations of $10^{\circ}, 20^{\circ}, 45^{\circ}$. 


\begin{tabular}{|c|c|c|c|c|}
\hline & lena & tree & water & baboon \\
\hline 0.9: detected triangle & $22 / 66$ & $3 / 64$ & $17 / 67$ & $5 / 66$ \\
\hline $0.9:$ global detection & $\mathrm{Ok}$ & $\mathrm{Ok}$ & $\mathrm{Ok}$ & $\mathrm{Ok}$ \\
\hline $0.7:$ detected triangle & $5 / 54$ & $0 / 52$ & $9 / 66$ & $0 / 44$ \\
\hline $0.7:$ global detection & $\mathrm{Ok}$ & Failure & $\mathrm{Ok}$ & Failure \\
\hline
\end{tabular}

Table 4: Robustness to scaling with factors of 0.9 and 0.7 .

\begin{tabular}{|c|c|c|c|c|}
\hline & lena & tree & water & baboon \\
\hline print and scan: detected triangle & $1 / 66$ & $0 / 64$ & $1 / 67$ & $2 / 64$ \\
\hline print and scan: global detection & $\mathrm{Ok}$ & Failure & Failure & Failure \\
\hline rotation $5^{\circ}:$ detected triangle & $0 / 68$ & $0 / 65$ & $1 / 68$ & $3 / 60$ \\
\hline rotation $5^{\circ}:$ global detection & $\mathrm{Ok}$ & Failure & Failure & Ok \\
\hline
\end{tabular}

Table 5: Robustness to print and scan process, the different results are given with and without the rotation of the printed sheet.

\section{Illustrations}




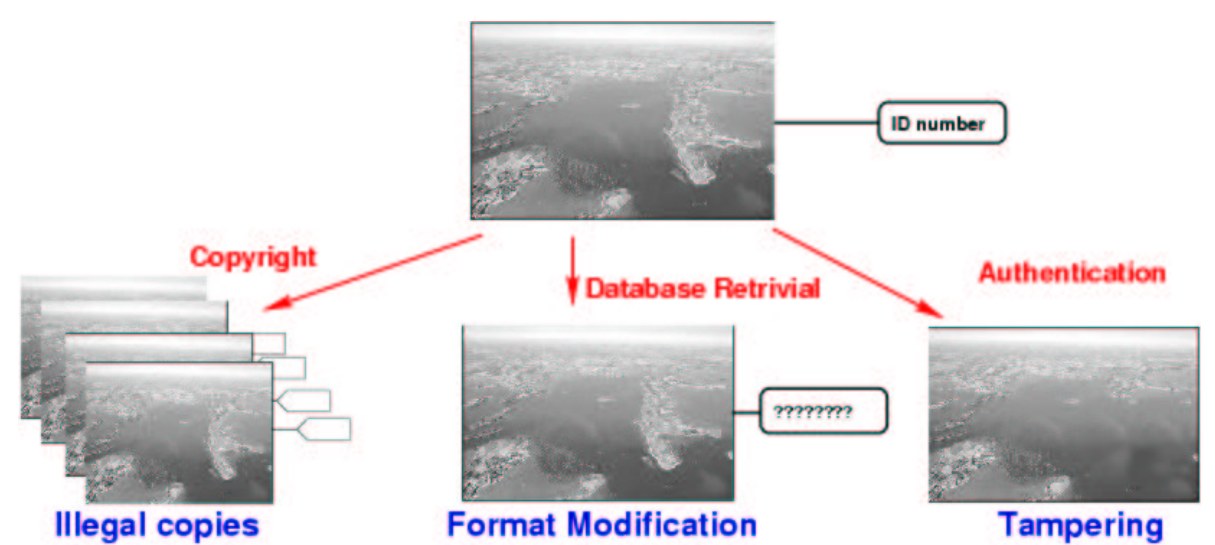

Figure 1: Illustration of watermarking applications: copyright, database retrieval and authentication
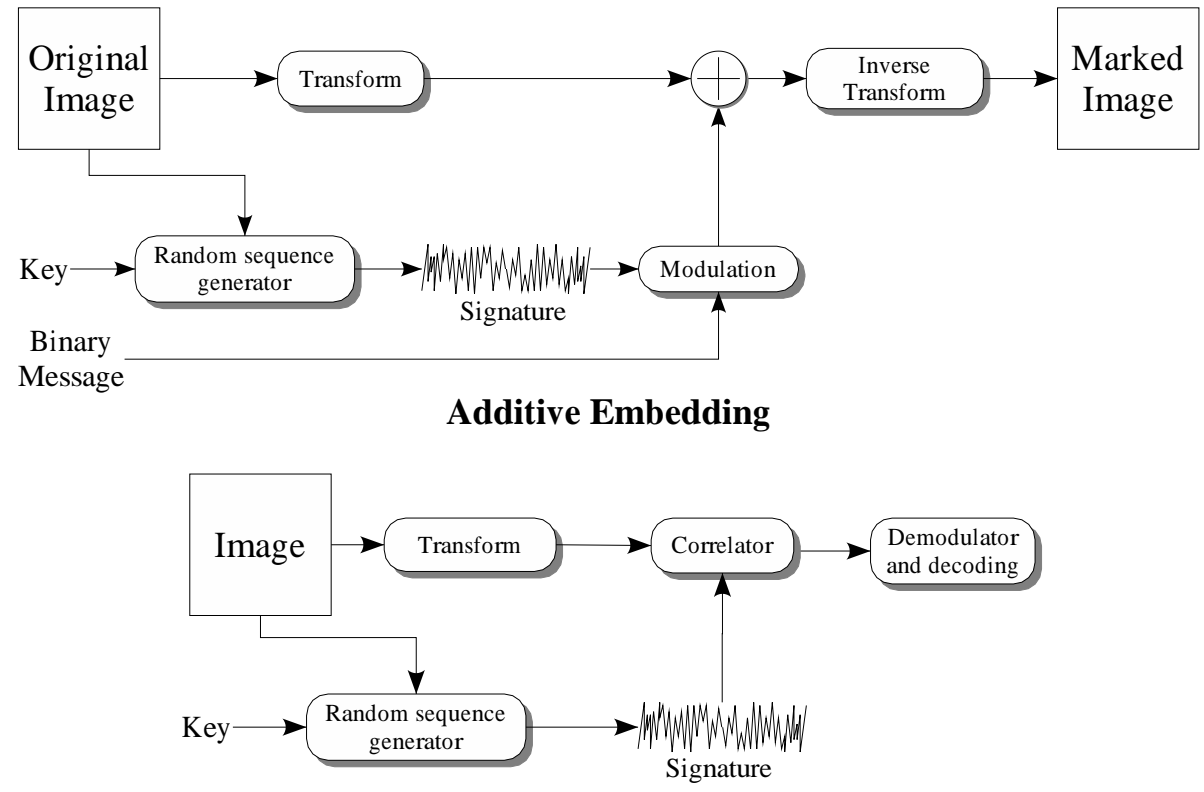

Additive Detection

Figure 2: Principle of embedding and detection of an additive scheme 


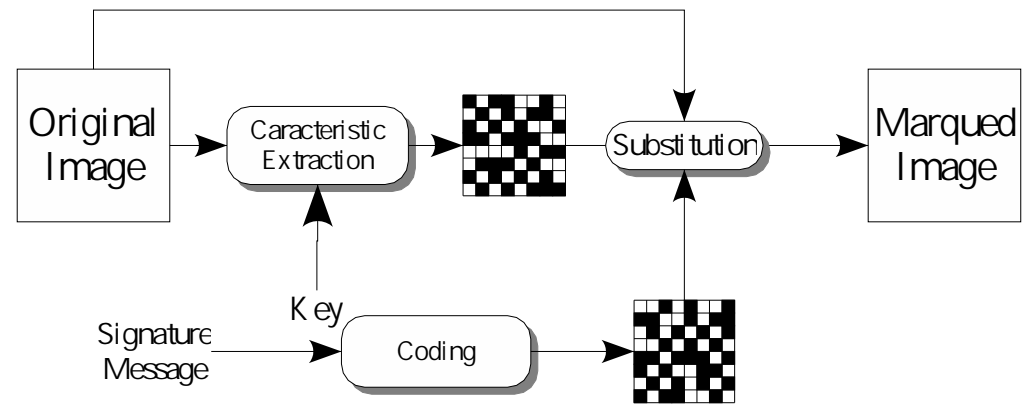

Substitutive Embedding

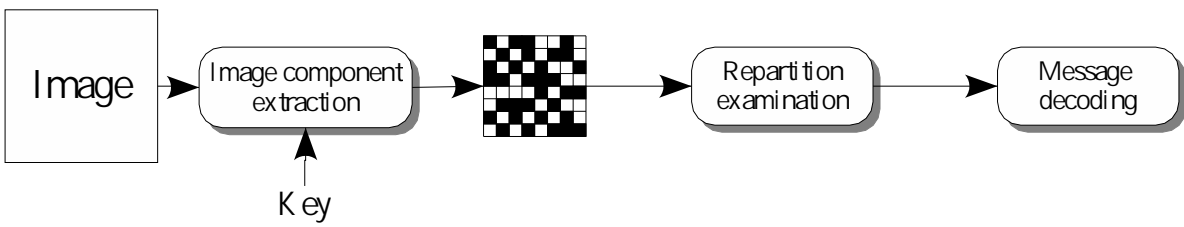

Substitutive Detection

Figure 3: Principle of embedding and detection of a substitutive scheme

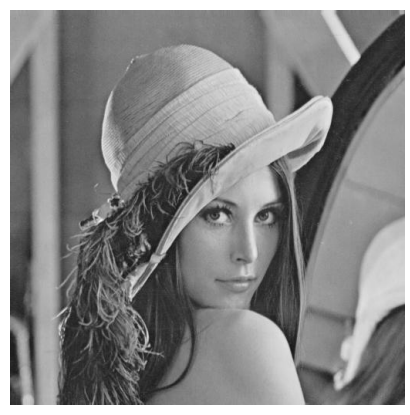

Initial Image

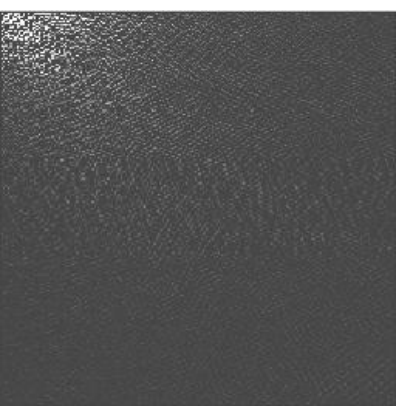

DCT coefficients

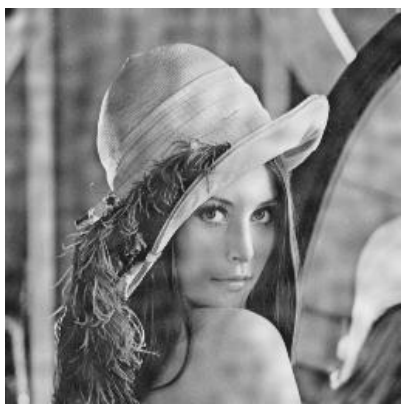

Increased mark

Figure 4: Frequential domain watermarking: the mark is embedded on DCT coefficient (middle figure) of the image (right figure) using a frequential scheme developed by Cox et al. The left figure represents a marked image with an increased mark. 


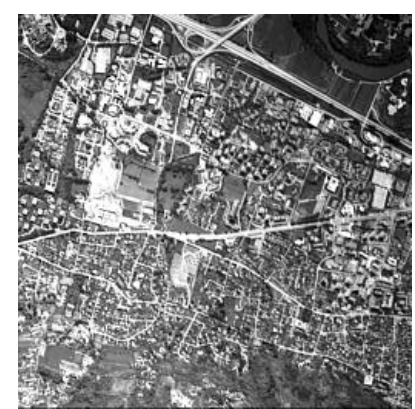

Initial image

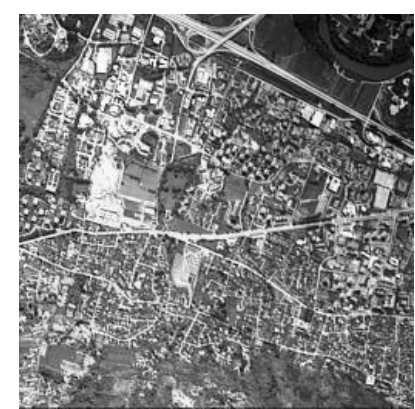

Marked Image

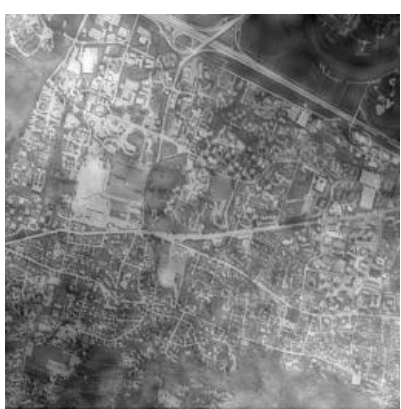

Marked Image

invisibility coeff $=0.06 \quad$ invisibility $\operatorname{coeff}=1.0$

Figure 5: Frenquential domain watermarking on an aerial image. Visibility effects

$$
\begin{aligned}
& \text { message to transmit: " LIS " } \\
& \qquad \begin{array}{|lll}
L \rightarrow 12 & I \rightarrow 9 & S \rightarrow 19 \\
\hline
\end{array}
\end{aligned}
$$
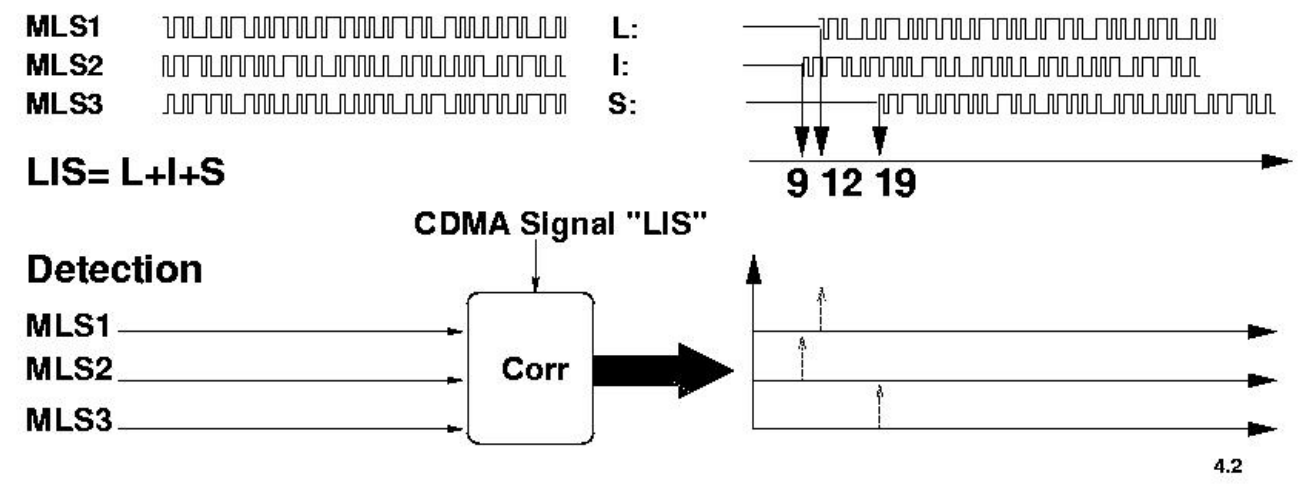

Figure 6: Illustration of generation of a sequence issued from message "LIS" using CDMA technique. 


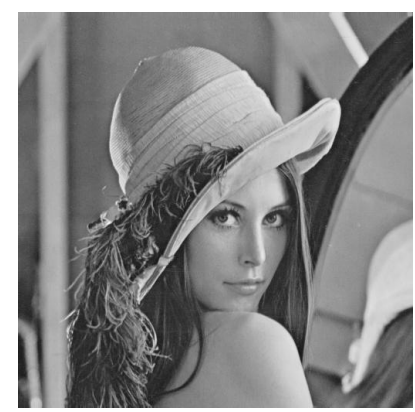

image 1

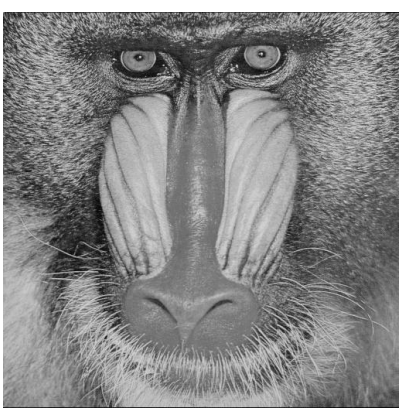

image 2

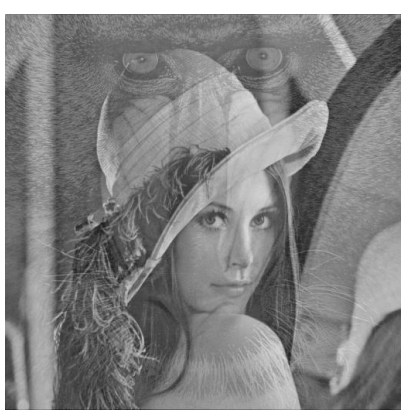

dissolved image

Figure 7: Example of the masking phenomena: the dissolved image is obtained adding $70 \%$ of image 1 and $30 \%$ of image 2 . We can see that textured region as better masking properties than homogeneous regions.

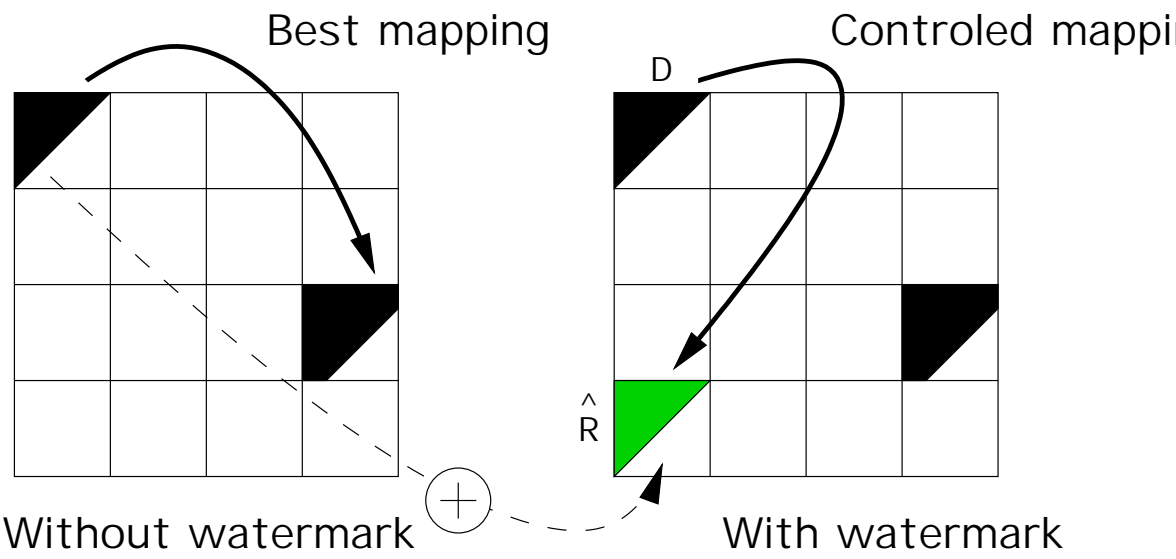

Figure 8: Image content dependent insertion using similarities: in this scheme the insertion of the mark is done by adding local similarities 


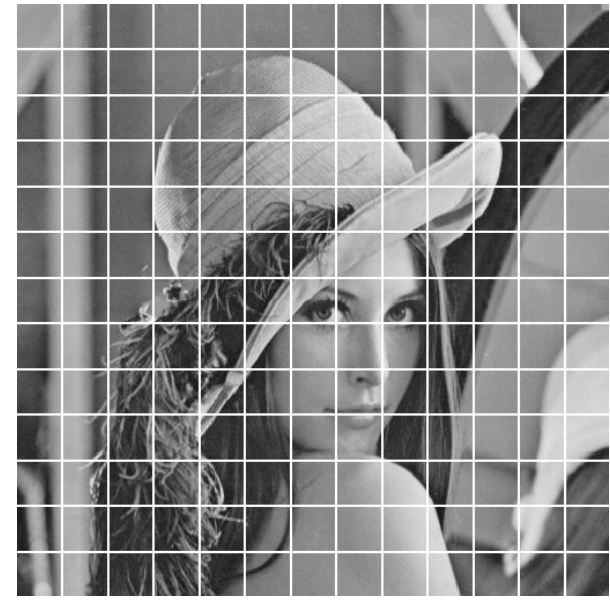

initial image

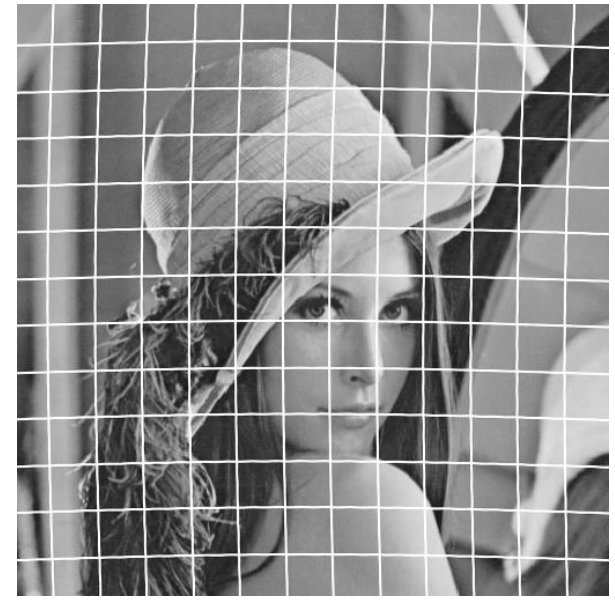

image after stirmark

Figure 9: Effect of the stirmark attack

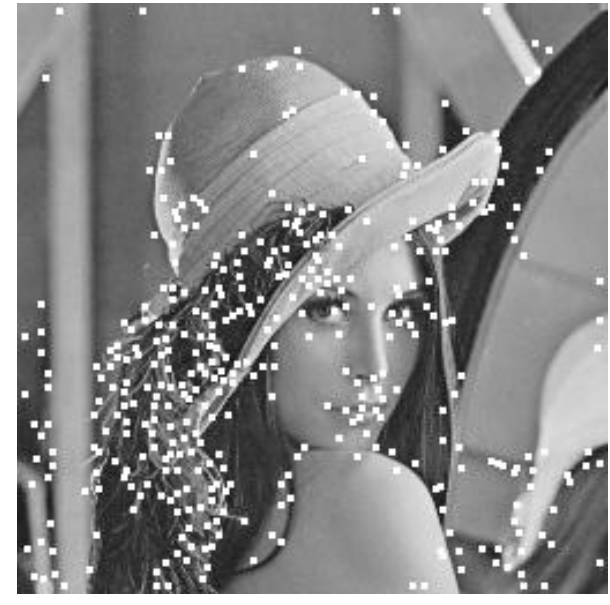

window $=4$

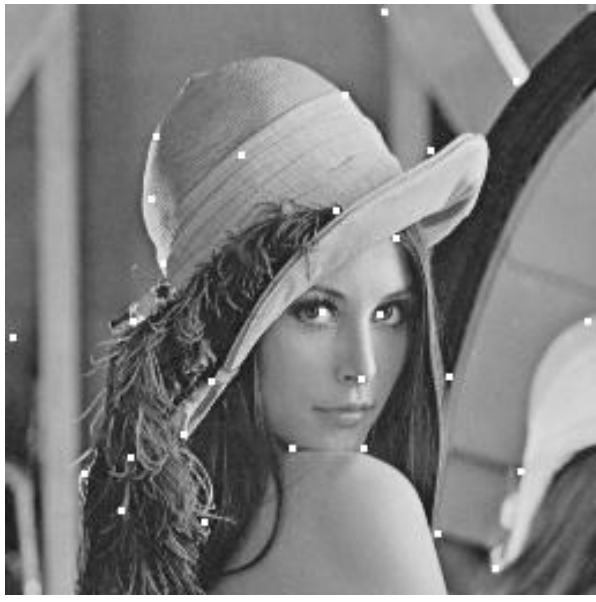

window $=40$

Figure 10: Feature point detection based on the Harris detector. The growth of the local maximum window permits to control the density of feature points. 


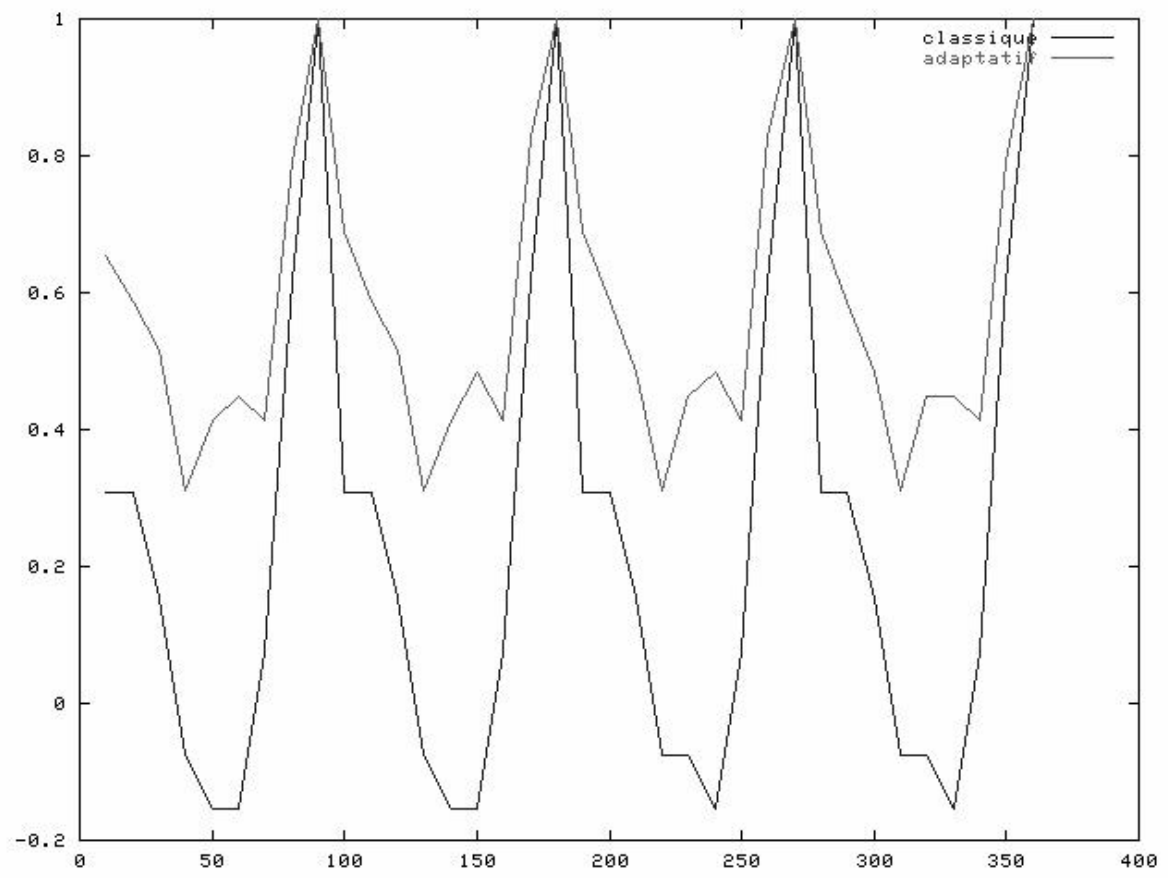

Figure 11: Comparison of the robustness of the harris detector after rotation. The $\mathrm{Y}$-axis represents the score of the detector and the $\mathrm{X}$-axis the angle in degree. We can see that our improved detector (upper plot) is more robust that the initial one (lower plot). 


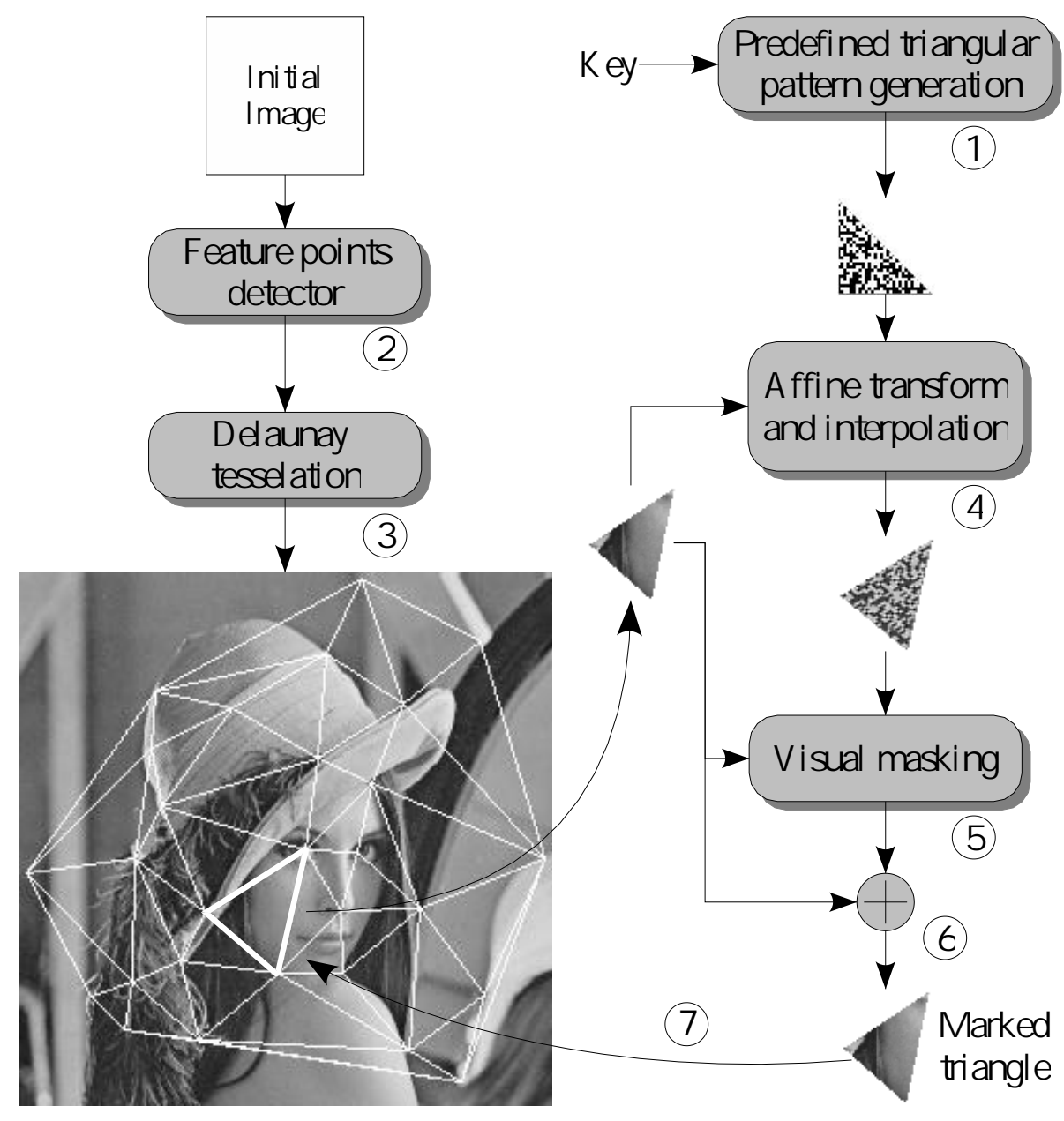

Figure 12: Embedding scheme based on the warping of pre-defined triangular patterns 


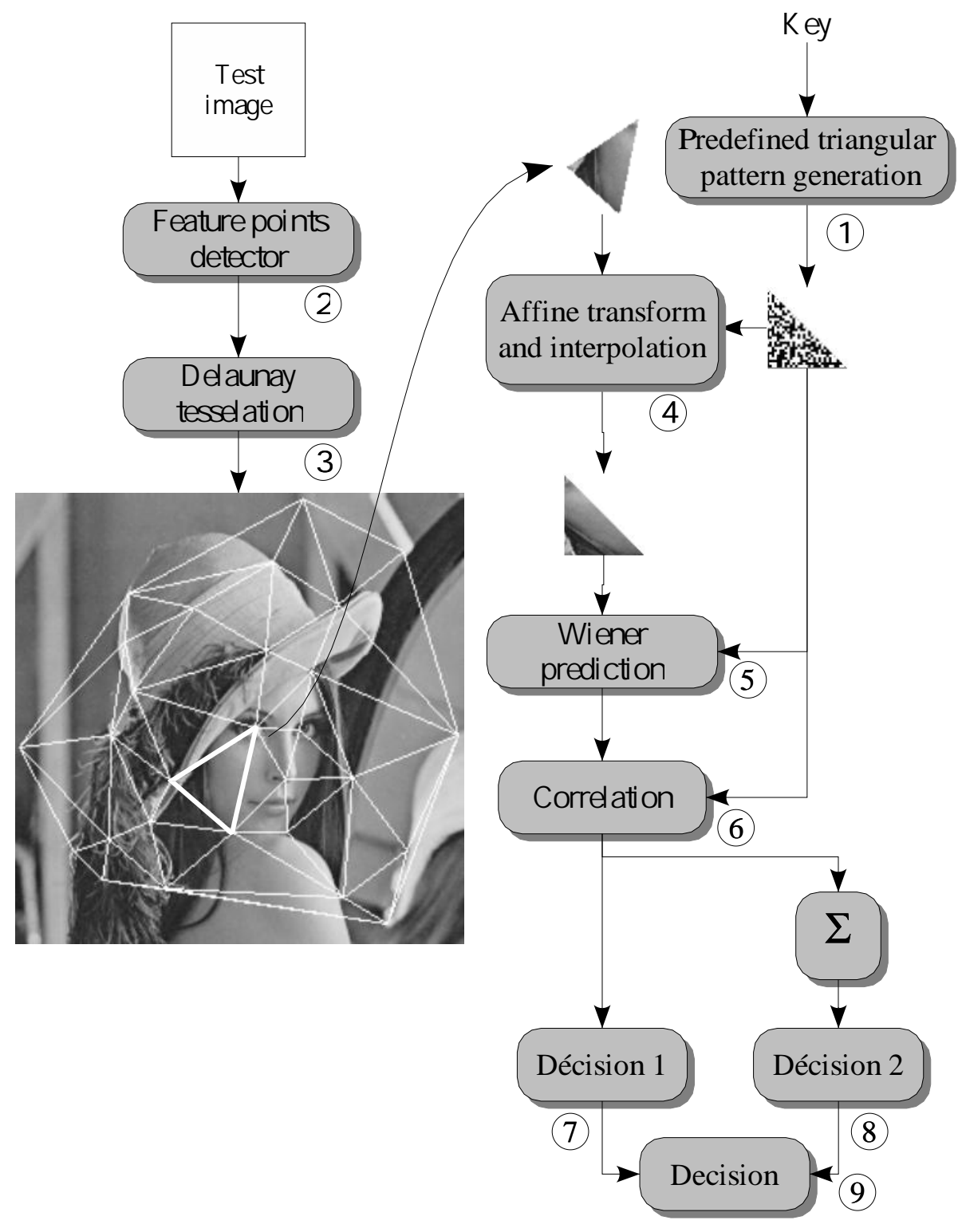

Figure 13: Detection scheme based on the warping of pre-defined triangular patterns 


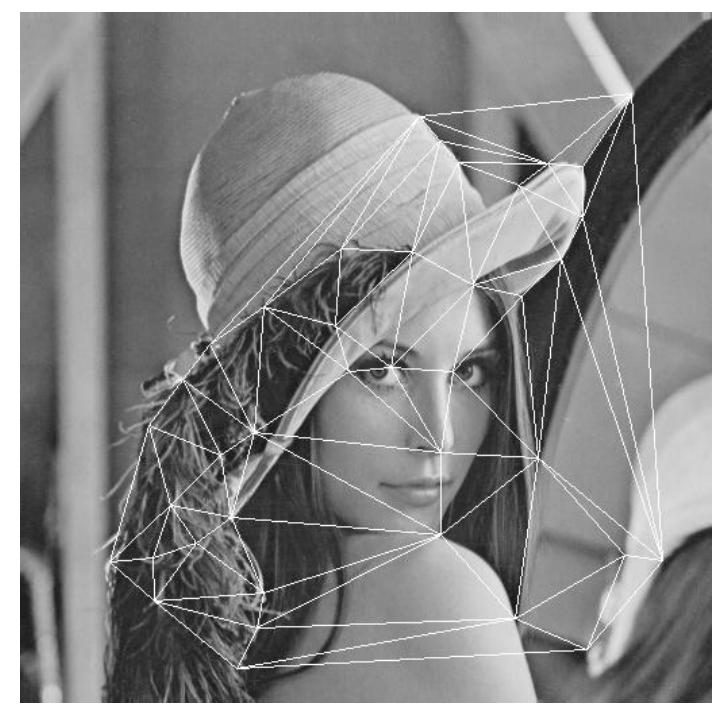

original image and tesselation

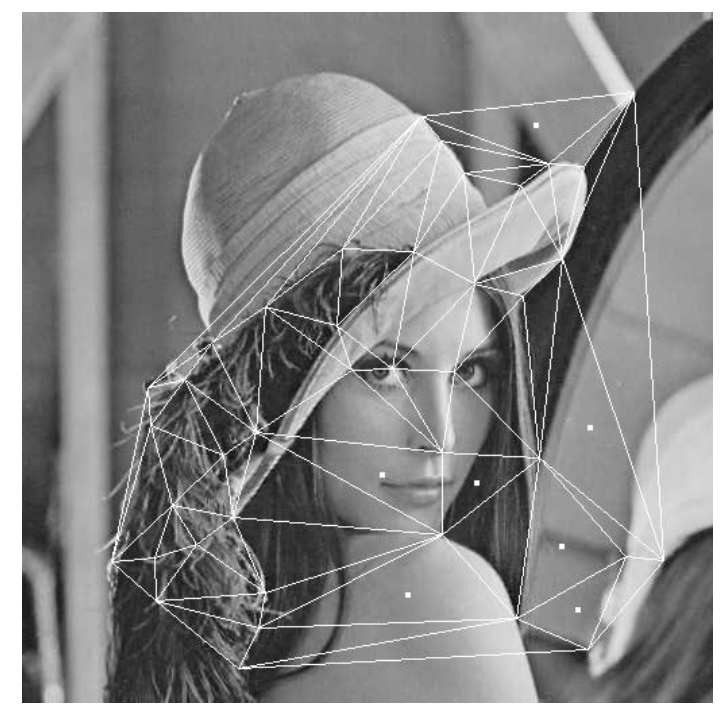

mark image and jpeg compression of $50 \%$

Figure 14: Jpeg compression on the "lena" image, the mark has been detected on 7 triangles of a tesselation of 68 triangles.

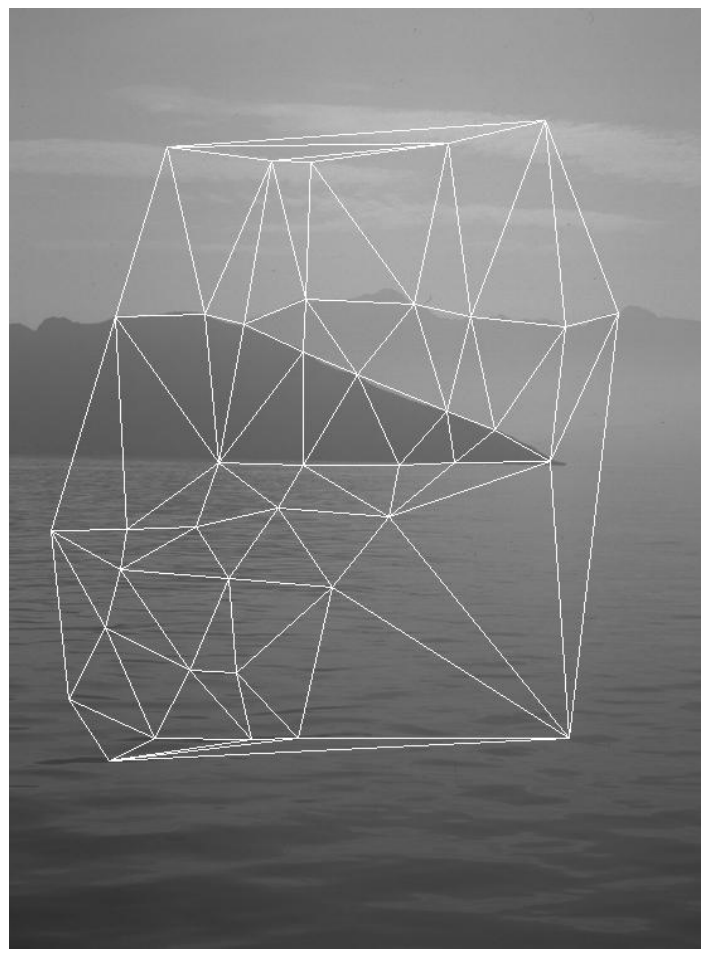

original image and tesselation

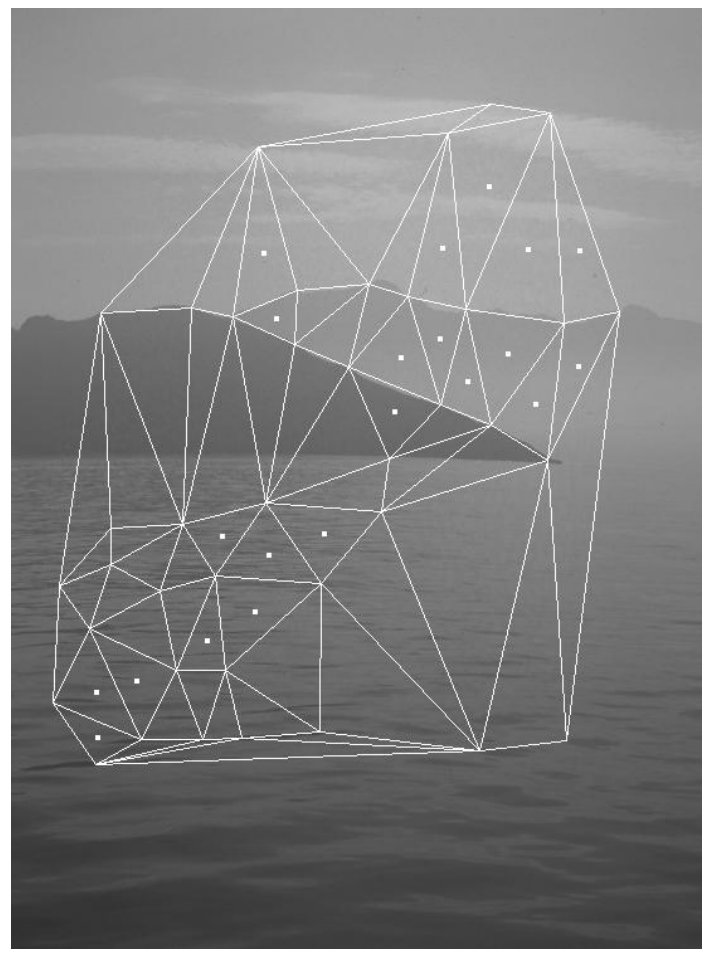

mark image after stirmark attack

Figure 15: Stirmark.3 attack on the image "water" ,the mark has been detected on 21 triangle of a tesselation of 65 triangles. 


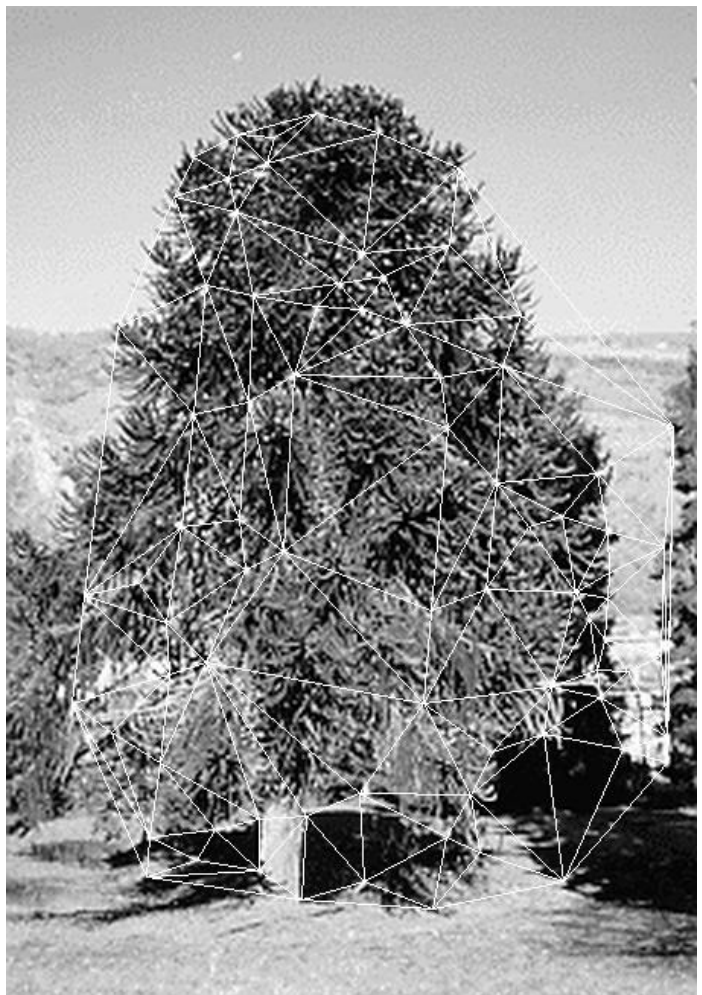

original image and tessellation

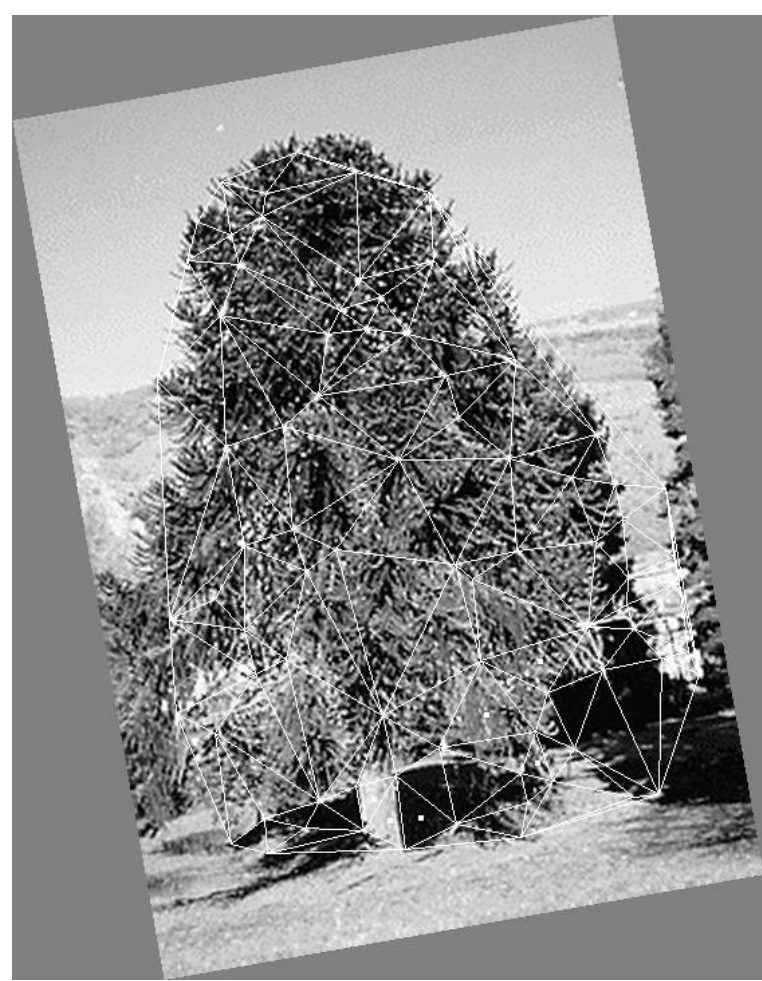

image tatoue aprs une rotation de $10^{\circ}$

Figure 16: Effect of a rotation of $10^{\circ}$ on the image tree, the mark has been detected on 6 triangle of a tessellation of 127 triangles. 


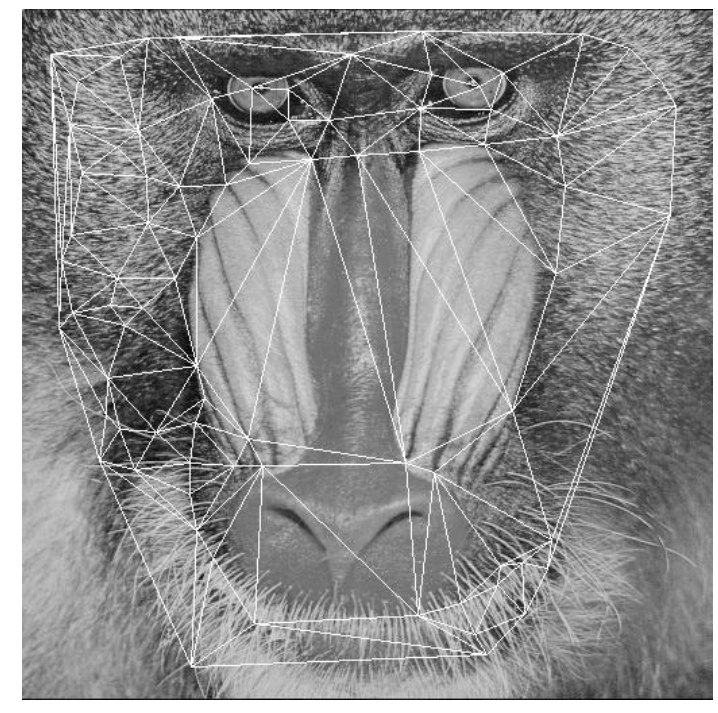

original image and tessellation

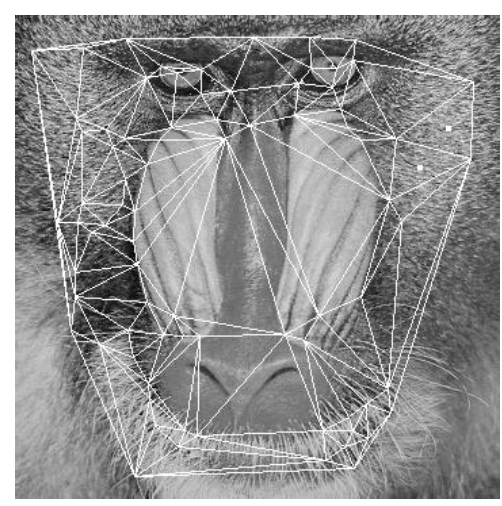

marked image

after a scaling factor of 0.7

Figure 17: Scaling effect with a factor of 0.7 on the baboon image, the mark have been detected on 2 triangles of a tessellation of 69 triangles.

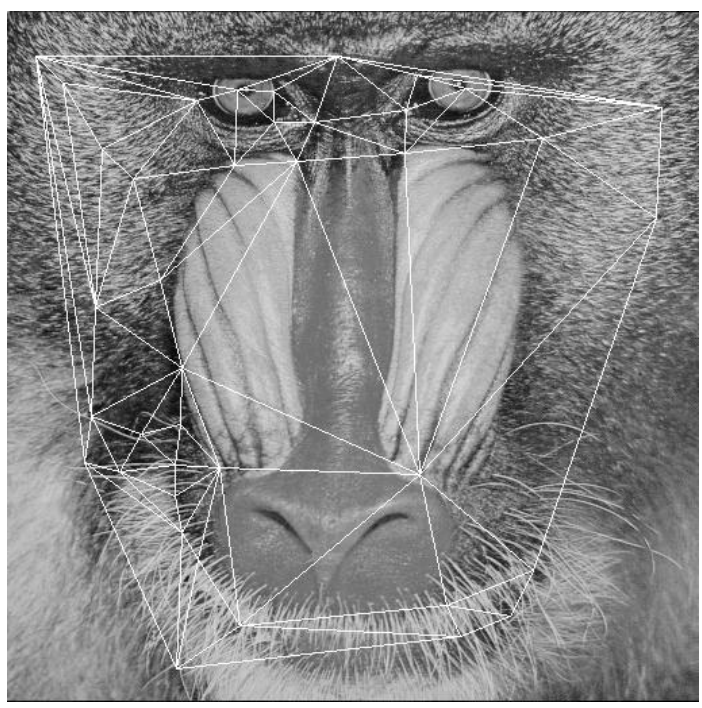

original image and tesselation

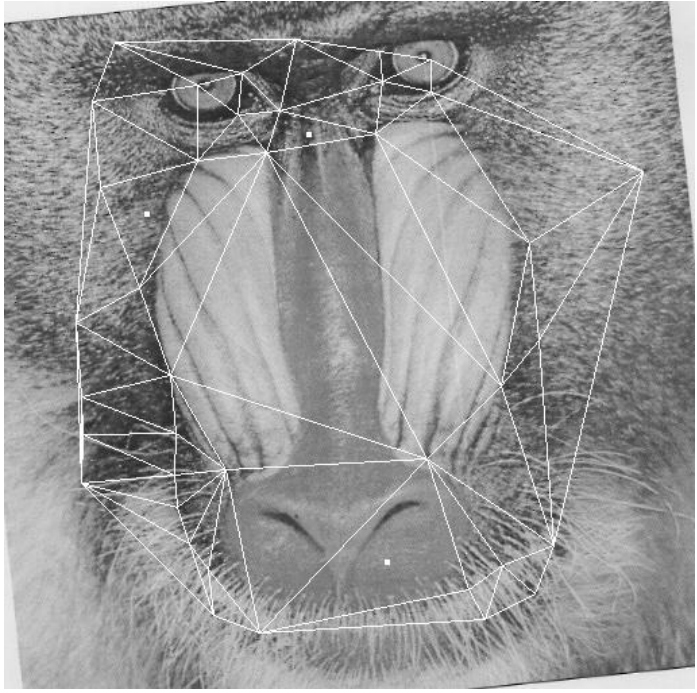

marked image

after "print and scan" process

Figure 18: Print and scan effect with a light rotation of the sheet: the mark is detected on 2 triangles of a tessellation of 60 triangles. 


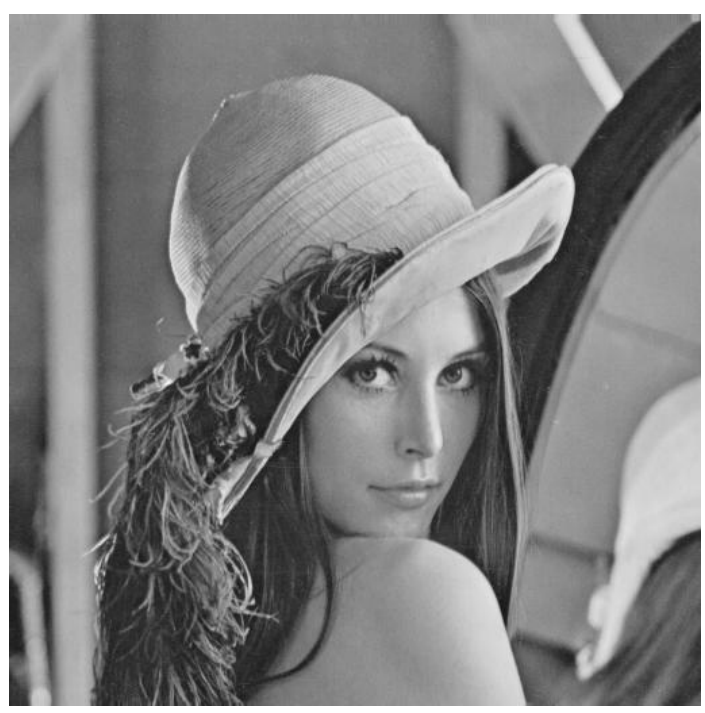

"lena" $512 \times 512$

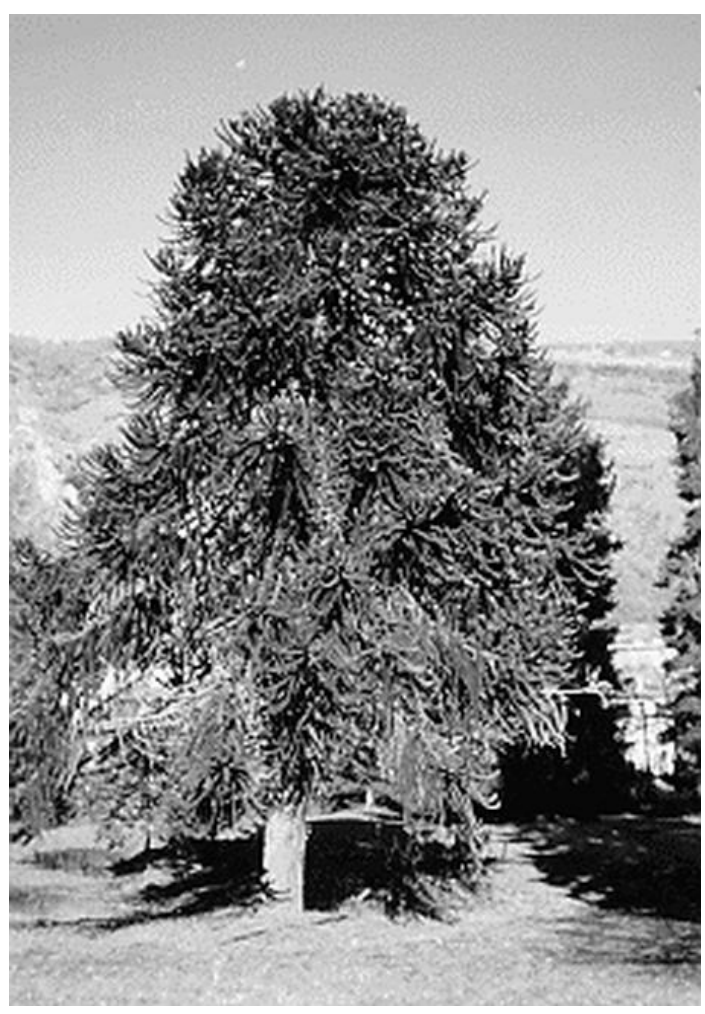

"tree" $454 \times 652$

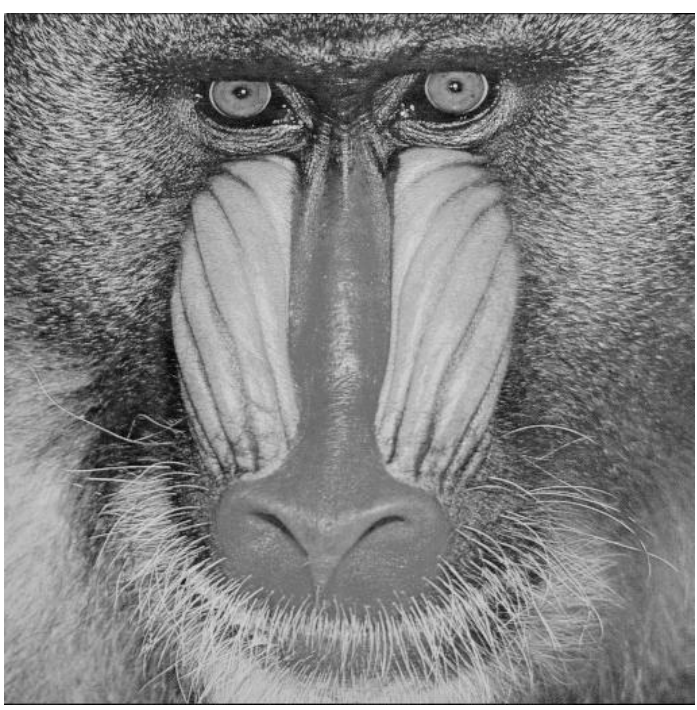

"baboon" $512 \times 512$

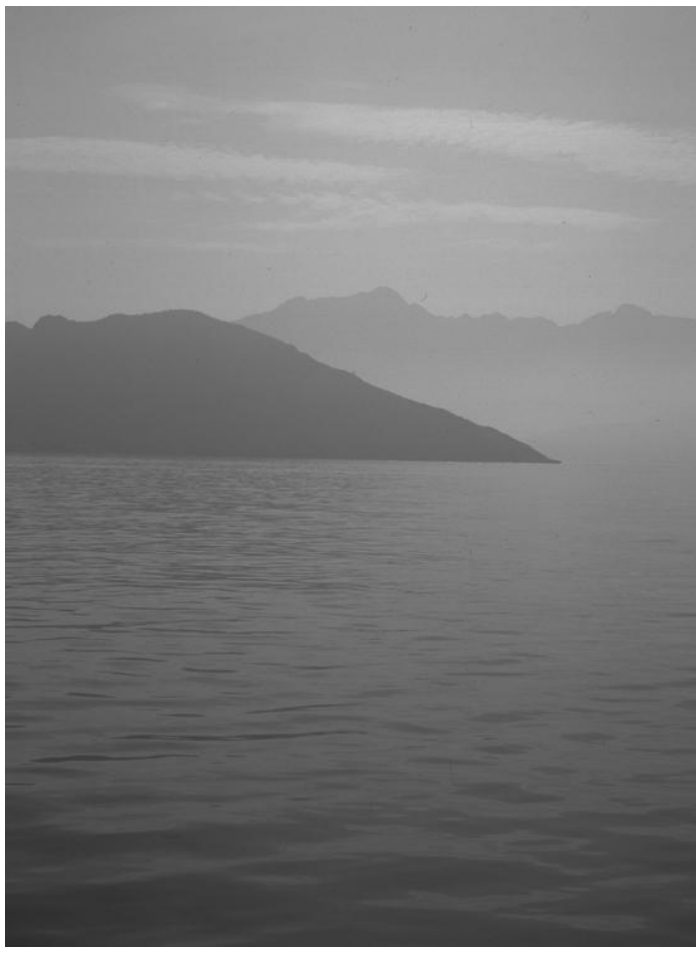

"water" $549 \times 748$

Figure 19: Different tested images. 